\title{
Predictor-Based Control of a Class of Time-Delay Systems and its Application to Quadrotors
}

\author{
Ricardo Sanz, Student Member, IEEE, Pedro Garc'ıa, Qing-Chang Zhong, Senior Member, IEEE, \\ and Pedro Albertos, Life Senior Member, IEEE
}

\begin{abstract}
In this paper, a new robust control strategy based on a predictor and the uncertainty and disturbance estimator (UDE) is developed for a class of uncertain nonlinear systems with input/output delays. The closed-loop system is analyzed and sufficient stability conditions are derived based on Lyapunov analysis. The proposed strategy is applied to the particular case of quadrotor systems and validated through extensive simulations to evaluate performance and robustness. The controller is also implemented in a quadrotor prototype and validated in flight tests.
\end{abstract}

Index Terms-Prediction, nonlinear systems, robust control, time-delay, uncertainty and disturbance estimator (UDE), quadrotor.

\section{INTRODUCTION}

$\mathrm{U}$ NMANNED Aerial Vehicles (UAVs) have gained an enormous interest for their civil potential applications.

Among different UAVs, quadrotors are remarkably popular and have been used extensively in research over the past decade [1], [2]. A high-performance attitude control is a prerequisite for developing any other high-level control tasks

[3]. The quadrotor dynamics involves challenges such as parametric uncertainties, non-linearity, coupling and external disturbances. Although many solutions have been proposed in the literature, very few of them have been validated in real flight tests and the most popular techniques are still based on classical control strategies [4]-[7]. This is mainly due to the constraints imposed by the limited computational resources of the embedded systems, which are typically micro-controllers. Also, and perhaps more importantly, because of the unstable nature of quadrotors, controllers must run typically at very high frequencies [8].

Manuscript received April 15, 2015; revised August 27, 2015, November 7, 2015, January 15, 2016, April 12, 2016 and June 24, 2016; accepted August 5, 2016. This work was partially supported by PROMETEOII/2013/004, Conselleria d'Educació , Generalitat Valenciana, and TIN2014-56158-C4-4-P-AR, Ministerio de Econom'la y Competitividad, Spain.

Ricardo Sanz, Pedro Garc'la and Pedro Albertos are with the Instituto de Automática e Informática Industrial, Universitat Politècnica de València, 46020 Valencia, Spain (e-mail: risanda@upv.es, pggil@isa.upv.es, pedro@aii.upv.es).

Qing-Chang Zhong is with the Department of Electrical and Computer Engineering, Illinois Institute of Technology, Chicago, IL 60616, USA (email: zhongqc@ieee.org).
Robust control for quadrotors is still an active field of research [9]-[11] because the aerodynamic effects are extremely

hard to be accurately modeled [12] and, specially in outdoor applications, a UAV is constantly perturbed by wind gusts [13]. Disturbance observers have drawn the attention of many researchers [10], [14], [15] as a tool for facing these problems.

Several approaches exist in the literature related to control based on the estimation of uncertainties and external disturbances, for example, adaptive robust control [16], uncertainty and disturbance estimator (UDE) [17], [18], extended state observer based control [19], disturbance observer based control (DOBC) [20], active disturbance rejection control (ADRC)

[21], etc. The UDE strategy has demonstrated remarkable performance in handling uncertainties and disturbances in practical applications [22]-[25].

Among the different problems that must be overcome in real implementations, time delays [26] deserve a special attention. In a micro-aerial vehicle, the angular position and velocity are typically estimated by means of filters resulting in delayed measurements [27]. Most control strategies can fail even for very small delays, which unavoidably appear in practical implementations due to the computational time, communications or actuator delays, mainly if fast disturbances are expected. In order to extend the applicability of these strategies to time delay systems, some modifications are needed.

The main contribution of this paper is to propose a new control strategy for a class of nonlinear time-delay systems, with a particular application to real-time quadrotor attitude control. The proposed control law combines a modified UDE with a state predictor that can be applied to control systems with measurement or actuation delays. The proposed method not only remains stable under the presence of large time delays, but it also results in a much better performance when small delays are present, as it is the case of any digital control system [28]. Sufficient conditions for the closed-loop stability are derived. The control law is validated through simulations and in real-time experiments with quadrotors.

The rest of the paper is structured as follows. The problem is formulated in Section II, where some preliminaries on the state prediction are also introduced. The proposed control law is developed in Section III and then the closed-loop stability is analyzed. In Section IV, the strategy is applied to the particular case of quadrotor systems, where extensive simulations illustrate the performance and robustness of the 
controller. The algorithm is also validated in an experimental laboratory platform, followed by flight tests in a quadrotor prototype. Some conclusions are drafted in the last section.

Notation: Along this paper, an $n$-dimensional vector is expressed as $\mathrm{x}=\left[\mathrm{x}_{1}, \ldots, x_{n}\right]^{T} \in \mathrm{R}^{n}$, and its 1-norm is simply denoted by "x" $=-{ }_{i=1}^{n}\left|x_{\boldsymbol{i}}\right|$. The induced 1-norm of an $m \times n_{\text {matrix }} \mathrm{X} \in \mathrm{R}^{m \times{ }^{\prime} \times} n_{\text {is analogously denoted by }} \mathrm{X}^{\prime \prime}=$

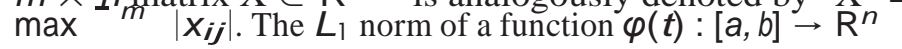
${ }_{\text {is }}^{j}$ represented by $" \varphi{ }_{1}={ }_{s}^{b} " \varphi(s) " \mathrm{~d} s$ while $" \varphi{ }^{\prime}{ }_{\infty}=$ $\sup _{s \in[a, b]} " \varphi(s) "$.

\section{Problem formulation ANd PREliminaries}

Consider the following class of nonlinear systems

$$
\begin{aligned}
& \dot{\mathrm{x}}(t)=A \mathrm{x}(t)+B\left[\mathrm{u}\left(t-h_{1}\right)+\mathrm{d}(t)\right]+\mathrm{f}(\mathrm{x}(t)) \\
& \mathrm{y}(t)=\mathrm{x}\left(t-h_{2}\right)
\end{aligned}
$$

where $\mathrm{x} \in \mathrm{R}^{n}$ and $\mathrm{u} \in \mathrm{R}^{m}$ are the state and control variables respectively, $\mathrm{f}: \mathrm{R}^{n} \rightarrow \mathrm{R}^{n}$ is an unknown possibly nonlinear function, and $d: R_{\geq 0} \rightarrow R^{m}$ is the vector of external disturbances. It is assumed that there is a constant input delay $h_{1}$, and also that the state of the plant is fully accessible with a measurement delay $h_{2}$. The total delay in the loop is denoted by $h=h_{1}+h_{2}$. A representation of such system is depicted in Fig. 1(a). The following assumptions are taken:

Assumption 1. The pair (A, B) is controllable

Assumption 2. The time delay $h \geq 0$ is constant and known

Assumption 3. The uncertainty $\mathrm{f}(\mathrm{x})$ belongs to the column space of $\mathrm{B}$, i.e., there exists a vector $\mathrm{d}_{f}(\mathrm{x}) \in \mathrm{R}^{m}$ such that $\mathrm{f}(\mathrm{x})=\mathrm{Bd}_{f}(\mathrm{x})$

Assumption 4. There is a region $\mathrm{D}=\left\{\mathrm{x} \in \mathrm{R}^{n}:\right.$ " $\left.\mathrm{x} " \leq r_{x}\right\}$ where: i.) $\mathrm{d}_{f}(\mathrm{x})$ is locally bounded, ii.) $\mathrm{d}_{f}(0)=0$ and iii.) its derivative is locally bounded by " $\nabla \mathrm{d} f(\mathrm{x}) " \leq c_{x}$

Assumption 5. The initial condition for (1) given by $\mathrm{x}(s)=\phi(s), \forall s \in[-h, 0]$ with $\phi:[-h, 0] \rightarrow \mathrm{R}^{n}$, is entirely contained in $\mathrm{D}$, that is, " $\phi{ }_{\infty}<\delta$ for some $\delta<r_{x}$

Assumption 6. The unforced system (1) starting from $\mathrm{x}(s)=\phi(s), \forall s \in[-h, 0]$ satisfies ${ }^{1} \mathrm{x}(\xi) "<\infty, \forall \xi \in[0, h]$

Assumption 7. The input disturbance $\mathrm{d}(t)$ is uniformly bounded and its derivative is bounded by $\|\dot{\mathrm{d}}(t)\| \leq c_{d}, \forall t \geq 0$

Let us define the lumped term $\mathrm{w}(\mathrm{x}(t), t) \in \mathrm{R}^{m}$, to contain all the model uncertainties and external disturbances as follows

$$
\mathrm{w}(\mathrm{x}(t), t) 3 / 4 \mathrm{~d}_{f}(\mathrm{x}(t))+\mathrm{d}(t) .
$$

Using (2) and the Assumption 3, the model (1) can be represented as shown in Fig. 1(b), that is

$$
\begin{aligned}
& \dot{\mathrm{x}}(t)=A \mathrm{x}(t)+B\left[\mathrm{u}\left(t-h_{1}\right)+\mathrm{w}(\mathrm{x}(t), t)\right] \\
& \mathrm{y}(t)=\mathrm{x}\left(t-h_{2}\right) .
\end{aligned}
$$

\footnotetext{
${ }^{1}$ For nonlinear systems that exhibit finite escape time, there is a limit above which the system cannot be controlled [29]. The Assumption 6 prevents the unforced system from exhibiting finite escape time smaller than $h$.
}

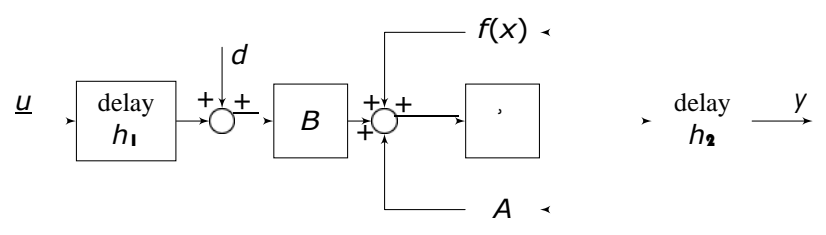

(a) Original system (1)

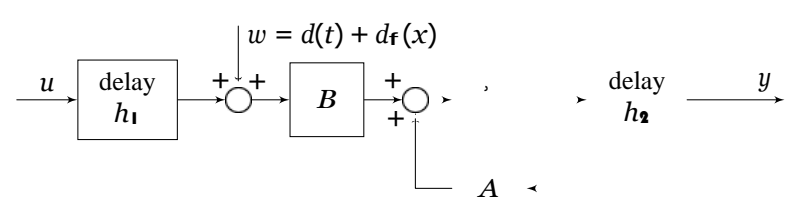

(b) Equivalent system (3)

Fig. 1. System representations

The underlying idea behind the original UDE [17] is that the unknown lumped signal $\mathrm{w}(\mathrm{x}(t), t)$ along the solutions of (3), denoted hereafter simply by $\mathrm{w}(t)$, can be accurately estimated and counteracted. However, as aforementioned, in the presence of input/output delays this strategy has limitations and needs to be improved. The extension of this methodology to time delay systems is presented in the next section. To this end, a conventional state prediction (see for example [30]) is computed by using the nominal model as

$$
\hat{\mathrm{x}}\left(t+h_{1}\right)=e_{\mathrm{A} h} \mathrm{x}\left(t-h_{2}\right)+{ }_{0}^{s} e_{\mathrm{A} \xi} \mathrm{Bu}(t-\xi) \mathrm{d} \xi .
$$

However, the model used to predict the state may be inaccurate because of model uncertainties or external disturbances, as stated in the following proposition.

Proposition 1. The error between the nominal prediction and the actual projection of the state is given by

$$
\mathrm{x}\left(t+h_{1}\right)-\hat{\mathrm{x}}\left(t+h_{1}\right)={ }^{h} e_{\mathrm{A} \xi} \mathrm{Bw}\left(t-\xi+h_{1}\right) \mathrm{d} \xi
$$

Proof. Using the actual model (3), the actual projected state is given by

$$
\begin{aligned}
\mathrm{x}\left(t+h_{1}\right) & =e^{\mathrm{A} h} \mathrm{x}\left(t-h_{2}\right)+{ }^{s} h e_{\mathrm{A} \xi} \mathrm{Bu}(t-\xi) \mathrm{d} \xi \\
& +{ }_{0}{ }^{h} e^{\mathrm{A} \xi} \mathrm{Bw}\left(t-\xi+h_{1}\right) \mathrm{d} \xi .
\end{aligned}
$$

The proposition follows subtracting (6) and (4).

\section{Proposed control strategy}

\section{A. Control lawdevelopment}

The goal is to regulate the state $\mathrm{x}(t)$ of the closed-loop system so that it asymptotically tracks the state of the reference model with the desired dynamics given by

$$
\dot{\mathrm{x}}_{m}(t)=\mathrm{A}_{m} \mathrm{x}_{m}(t)+\mathrm{B}_{m} \mathrm{r}\left(t-h_{1}\right),
$$

where $\mathrm{A}_{m} \in \mathrm{R}^{n \times n}$, is Hurtwitz, $\mathrm{B}_{m} \in \mathrm{R}^{n \times m}, \mathrm{x}_{m} \in \mathrm{R}^{n}$ and $\mathrm{r}(t) \in \mathrm{R}^{m}$. 


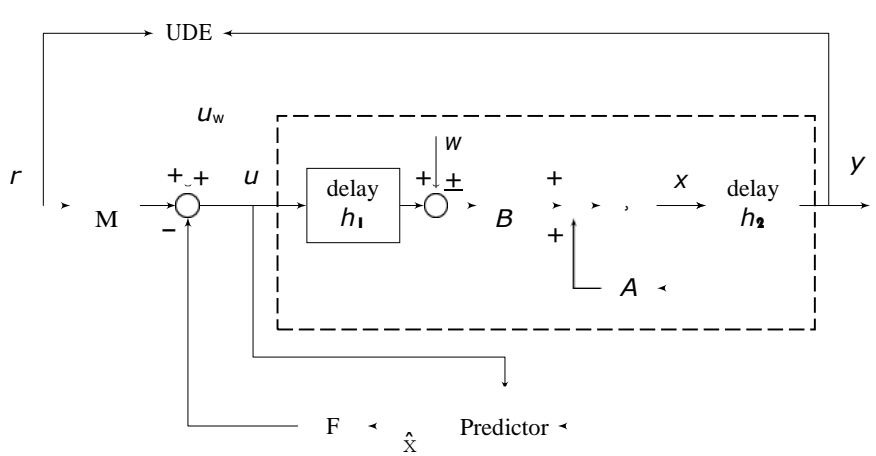

Fig. 2. Proposed control strategy

Assumption 8. The input reference command $\mathrm{r}(t)$ is bounded by $" \mathrm{r}(t) " \leq \rho, \forall t \geq 0$

Now, the following feedback law is proposed (see Fig. 2)

$$
\mathrm{u}(t) 3 / 4-\mathrm{F} \hat{\mathrm{x}}\left(t+h_{1}\right)+\mathrm{Mr}(t)+\mathrm{u}_{w}(t),
$$

where the matrices are chosen such that

$$
\mathrm{A}-\mathrm{BF}=\mathrm{A}_{m}, \quad \mathrm{BM}=\mathrm{B}_{m},
$$

and the term $\mathrm{u}_{w}(t)$, defined further below, will be used to compensate the uncertainties. Introducing (8) into the system (3) and using (9) yields

$$
\begin{aligned}
& \dot{\mathrm{x}}(t)=\mathrm{A}_{m} \mathrm{x}(t)+\mathrm{B}_{m} \mathrm{r}\left(t-h_{1}\right)+B\left[\mathrm{u}_{w}\left(t-h_{1}\right)+\Delta(t)\right] \\
& \mathrm{y}(t)=\mathrm{x}\left(t-h_{2}\right),
\end{aligned}
$$

where the term

$$
\Delta(t) 3 / 4 \mathrm{w}(t)+\mathrm{F}_{0}^{s} e^{\mathrm{A} \xi} \mathrm{Bw}(t-\xi) \mathrm{d} \xi
$$

has been introduced to gather the original uncertainties of the system along with the error introduced by the predictor, both of them unknown. Once the effect of the delay has been counteracted, an observer based on the UDE is adopted in an outer loop to handle the overall uncertainties and disturbances. This approach might resemble the one-loop-at-a-time design procedure widely used in aircraft control, where an inner loop referred to as stability augmentation system (SAS) is used to increase stability, and outer loops are used to provide additional features for maneuvering [31].

The new control input $u_{w}(t)$ should be chosen to cancel out the term $\Delta(t)$ which, despite being unknown, can be expressed using (10) as

$$
\Delta(\mathrm{t})=\mathrm{B}^{+}\left[\dot{\mathrm{x}}(t)-\mathrm{A}_{m} \mathrm{x}(t)-\mathrm{B}_{m} \mathrm{r}\left(t-h_{1}\right)-B \mathrm{u}_{w}\left(t-h_{1}\right)\right] .
$$

Equation (12) is not implementable because the state $x(t)$ is not accessible at time $t$. Instead of (12), consider the signal

$$
\Delta\left(t-h_{2}\right)=B^{+}\left[\dot{y}(t)-A_{m} y(t)+B_{m} r(t-h)-B u_{w}(t-h)\right],
$$

which is the result of delaying $\Delta(t)$ by $h_{2}$ units of time. In this way, the measurement $\mathrm{y}(t)=\mathrm{x}\left(t-h_{2}\right)$ appears in the signal to be estimated. Another handicap is that the term $\dot{\mathrm{y}}(t)$ is not realizable, but it can be approximated in the frequency domain by using a strictly-proper low-pass filter $\left.{ }^{2} t t_{f}(s)=1 / T_{f} S+1\right)$ [17]. Then, the estimated uncertainty can be defined as

$$
\hat{\Delta}\left(t-h_{2}\right) 3 / 4 \mathrm{~L}^{-1}\left\{\mathrm{G}_{f}(s)\right\} * \Delta\left(t-h_{2}\right),
$$

where $\mathbf{G}_{f}(s)=t t_{f}(s) \mathrm{I}_{m}$. And thus, selecting

$$
\mathrm{u}_{w}(t) 3 / 4-\hat{\Delta}\left(t-h_{2}\right)
$$

and plugging it into (10) results in the closed-loop system

$$
\dot{\mathrm{x}}(t)=\mathrm{A}_{m \mathrm{x}}(t)+\mathrm{B}_{m} \mathrm{r}\left(t-h_{1}\right)+\mathrm{Be}(t)
$$

where $\mathrm{e}(t)$ is the cancellation error defined as

$$
\mathrm{e}(t) 3 / 4 \Delta(t)-\hat{\Delta}(t-h)
$$

Remark 1. In the nominal disturbance-free case $\mathrm{e}(t) \equiv 0$ and thus the closed-loop system (16) has the desired dynamics specified by the reference model (7).

In order to analyze the stability in the presence of uncertainties, the dynamics of the error (17) has to be derived. According to (14), the estimator dynamics can be expressed as

$$
\dot{\hat{\Delta}}(t-h)=-\frac{1}{T_{\boldsymbol{f}}} \hat{\Delta}(t-h)+\frac{1}{T_{\boldsymbol{f}}} \Delta(t-h)
$$

Differentiating (17), using (18), and adding and subtracting $\frac{1}{T_{f}} \Delta(t)$, the dynamics of the cancellation error can be written as

$$
\dot{\mathrm{e}}(t)=-\frac{1}{T_{f}} \mathrm{e}(t)+\mathrm{g}(t)
$$

where

$$
\mathrm{g}(t) 3 / 4 \dot{\Delta}(t)+\frac{1}{T_{f}}[\Delta(t)-\Delta(t-h)] .
$$

The initial condition for (19) is $\mathrm{e}(s)=\Delta(s), \forall s \in[-h, 0]$ by (17), assuming that the observer starts from zero, that is $\hat{\Delta}(s)=0, \forall s \in[-h, 0]$.

The closed-loop system is thus composed of (16) and (19), which can be expressed altogether as

$$
\dot{\eta}(t)=\mathrm{A}_{\eta} \eta(t)+\cdot{ }^{-}(t){ }^{-}+{ }^{-} \mathrm{Bom} \cdot \mathrm{r}\left(t-h_{1}\right),
$$

where $\eta(t)=[\mathrm{x}(t), \mathrm{e}(t)]^{T}$ is an augmented state and the matrix $A_{\eta}$ is given by

$$
\mathrm{A}_{\eta}={ }^{\circ} \mathrm{A}_{m}-\frac{\mathrm{B}_{\mathrm{I}_{\boldsymbol{f}}}}{} \cdot
$$

\section{B. Closed-loop stability}

As $A_{\eta}$ is Hurtwitz, the main issue to analyze the stability is the boundedness of the term $\mathrm{g}(t)$ that drives the error dynamics. This is addressed by the following lemma.

Lemma 1. Under Assumptions 4 and 7 , the term $\mathrm{g}(t)$ defined in (20) satisfies $" \mathrm{~g}(t) " \leq \gamma_{x} " \mathrm{x}(t) "+\gamma_{e} " \mathrm{e}(t) "+\gamma_{0}, \forall \mathrm{x} \in \mathrm{D}$, with constants $Y_{x}, Y_{e}, Y_{0}$ subsequently defined.

\footnotetext{
${ }^{2}$ The Laplace transformation is introduced to facilitate the manipulation of expressions.
} 
Proof. Let us first introduce the following notation $\alpha_{m} 3 / 4$ "A $m^{\prime}, \beta_{m} 3 / 4 \quad$ "B $m^{\prime}$ and $\beta \quad 3 / 4 \quad$ "B". According to the definition (20), $\mathrm{g}(t)$ can be bounded as

$$
" g(t) " \leq \dot{\Delta}(t)+\frac{1}{T_{f}} " \Delta(t)-\Delta(t-h) " .
$$

The first term in (22) is obtaine, by differentiating (11) as

$$
\begin{aligned}
\dot{\Delta}(t) & =\nabla \mathrm{d}_{\boldsymbol{f}} \cdot \dot{\mathrm{x}}(t)+\mathrm{F}{ }_{0} \mathrm{e}^{\mathrm{A}} \mathrm{B} \nabla \mathrm{d}_{\boldsymbol{f}} \cdot \dot{\mathrm{x}}(t-\xi) \mathrm{d} \xi \\
& +\dot{\mathrm{d}}(t)+\mathrm{F}_{0}{ }^{\mathrm{e}^{\mathrm{A} \xi} \mathrm{B}} \dot{\mathrm{d}}(t-\xi) \mathrm{d} \xi .
\end{aligned}
$$

By Assumptions 4,7, and using the Young's inequality for convolutions, that is $\phi * \varphi{ }^{\prime \prime}{ }_{1} \leq " \phi_{1} " \varphi_{\infty}$, , (23) can be bounded by

$$
\begin{aligned}
\dot{\Delta}(t) & \leq c_{x} " \dot{\mathrm{x}}(t) "+\mu c_{x} \sup _{\xi \in[t-h, t]} " \dot{\mathrm{x}}(\xi) " \\
& +c_{d}+\mu c_{d},
\end{aligned}
$$

where $\mu 3 / 4$ "F" ${ }^{-}{ }^{h} \quad e^{\mathrm{A} \xi} \mathrm{d} \xi \bar{\beta} \beta$. From (16), one has that $" \dot{\mathrm{x}}(t) " \leq \alpha_{m} " \mathrm{x}(t) "+\beta " \mathrm{e}(t) "+\beta_{m} \rho$. Assume now that there exist $q_{e}, q_{x}>1$ such that $" \mathrm{x}(t+\xi) " \leq q_{x}{ }^{\mathrm{x}}(t)$ " and "e $(t+\xi) " \leq q_{e} " \mathrm{e}(t) " \forall \xi \in[-h, 0]$, [32]. Note that this assumption does not imply a priori the stability of the system. Then $\sup _{\xi \in[t-h, t]} " \dot{\mathrm{x}}(\xi) " \leq \alpha_{m} q_{x} " \mathrm{x}(t) "+\beta q_{e} " \mathrm{e}(t) "+\beta_{m} \rho$. And thus (24) is finally bounded by

$$
\begin{aligned}
\dot{\Delta}(t) & \leq c_{x} \alpha_{m}\left(1+\mu q_{x}\right) " \mathrm{x}(t) "+c_{x} \beta\left(1+\mu q_{e}\right) " \mathrm{e}(t) " \\
& +(1+\mu)\left(c_{d}+c_{x} \beta_{m} \rho\right) .
\end{aligned}
$$

The second term in (22) can be bounded using the LeibnizNewton formula and the Young's inequality as follows

$$
\begin{aligned}
\frac{1}{T_{\boldsymbol{f}}} " \Delta(t)-\Delta(t-h) " & \leq{\frac{1}{T_{f}}}^{s t} \Delta(\xi) \mathrm{d} \xi \\
& \leq \frac{h}{T_{f}} \sup _{\xi \in[t-h, t]} \Delta(\xi) .
\end{aligned}
$$

Proceeding similarly as above, from (25) it is easy to obtain that

$$
\begin{array}{ll}
\sup _{\xi \in[t-h, t]} \dot{\Delta}(\xi) & \leq c_{x} \alpha_{m}\left(1+\mu q_{x}\right) q_{x} " \mathrm{x}(t)^{\prime \prime} \\
& +c_{x} \beta\left(1+\mu q_{e}\right) q_{e} " \mathrm{e}(t) " \\
& +(1+\mu)\left(c_{d}+c_{x} \beta_{m} \rho\right) .
\end{array}
$$

Gathering (22) and (25)-(27) yields

$$
\begin{aligned}
" \mathrm{~g}(t) " & \leq c_{x} \alpha_{m}\left(1+\mu q_{x}\right) 1+\frac{h q_{x}}{T_{f}} " \mathrm{x}(t) " \\
& +c_{x} \beta\left(1+\mu q_{e}\right) 1+\frac{h q_{e}}{T_{f}} " \mathrm{e}(t) " \\
& +\left(c_{d}+c_{x} \beta_{m} \rho\right)(1+\mu) 1+\frac{h^{-}}{T_{f}} \\
& 3 / 4 \gamma_{x} " \mathrm{x}(t)^{\prime}+\gamma_{e} \mathrm{e}(t) "+\gamma_{0} .
\end{aligned}
$$

This allows stating the following result:
Theorem 1. Under Assumptions 1-6, the system (1) having no external inputs, i.e, $\mathrm{d}(t)=\mathrm{r}(t) \equiv 0$, controlled by (8) is asymptotically stable for some $\delta>0$ and for any delay $0 \leq h \leq h^{*}$ if there exist a positive definite symmetric matrix

$\mathbf{P}$ such that $\mathbf{P A}_{\eta}+\mathbf{A}_{\eta} \mathbf{P}=-I_{m+n}$ and positive constants $q_{x}, q_{e}>1$ such that $2 . " \mathrm{P}_{2} "\left(\gamma_{x}+V_{e}\right)<1$ where $y_{x}=$ $c_{x} \alpha_{m}\left(1+\mu q_{x}\right)_{3} h^{1+} h^{*} q_{x}, Y_{e}=c_{x} \beta\left(1+\mu q_{e}\right) 1+{ }^{h q_{e}}$ and $\mu=" \mathrm{~F}^{*} e^{\mathrm{A}^{T^{F}}} \mathrm{~d} \xi \beta$.

0

Proof. Let us choose the Lyapunov candidate function $V(\eta)=\eta^{T} P \eta$ whose derivative along the trajectories of $(21)$, with $\mathrm{r}(t) \equiv 0$, is given by

$$
\begin{gathered}
\dot{V}(\eta)=\eta^{T}\left(\mathrm{PA}_{\eta}+\mathrm{A}_{\eta}^{T} \mathrm{P}\right) \eta+2 \eta^{T} \mathrm{P}_{2} \mathrm{~g}(t) \\
\leq-" \eta^{2}+2 " \eta "{ }^{2} \mathrm{P}_{2} " \text { "g" } .
\end{gathered}
$$

Setting $\mathrm{d}(t)=\mathrm{r}(t) \equiv 0$ in Lemma 1 , that is $c_{d}=\rho=0$, the term $V_{0}$ vanishes and, provided that $V_{x}, V_{e}>0$, then $" \mathrm{~g}(t) " \leq$ $\gamma_{x}{ }^{\mathrm{x}}(t) "+\gamma_{e} \mathrm{e}(t) " \leq\left(\gamma_{x}+\gamma_{e}\right) " \eta(t) "$. It follows then that for any " $\eta$ " $<r_{x}$ (which jmplies $\left.x \in D\right),(29)$ is bounded by

$$
\begin{aligned}
V(\eta) & \leq-" \eta^{\prime 2}+2 " \mathrm{P}_{2}\left(\gamma_{x}+\gamma_{e}\right) " \eta^{\prime \prime} \\
& \leq 0, \quad \text { if } \quad 2 " \mathrm{P}_{2} "\left(\gamma_{x}+\gamma_{e}\right)<1,
\end{aligned}
$$

and thus the system is asymptotically stable for $h^{*}$ if 2 " $\mathrm{P}_{2} "\left(\gamma_{x}+\gamma_{e}\right)<1$ hold. Note that $V(t)$ is always decreasing because its derivative is negative in " $\eta "<r_{x}$ by (30), and hence " $\eta(t)$ " is always decreasing because $V(\eta)=" P "{ }^{\prime \prime} \eta^{2}$. That implies that $\lim _{t \rightarrow \infty} " \eta(t) "=0$ if $" \eta(0) "<r_{x}$, which can always be achieved by choosing a small enough $\delta$ in Assumption 5. Furthermore, the terms $\gamma_{x}, \gamma_{e}$ grow monotonically with the delay an hence any $0 \leq h \leq h^{*}$ will also satisfy the condition, which completes the proof.

Corollary 1. If there is no delay, that is $h=0$, it is always possible to find a choice for $\mathrm{A}_{m} \prec 0, T_{\boldsymbol{f}}>0$ such that the system is asymptotically stable.

Proof. Setting $h=0$, the constants in Lemma 1 are simplified to $\gamma_{x}=c_{x} \alpha_{m}$ and $\gamma_{e}=c_{x} \beta$, and thus the stability condition of Theorem 1 is simply given by 2 " $\mathrm{P}_{2} " c_{x}\left(\beta+\alpha_{m}\right)<1$. Note that $\mathrm{P}$ is the solution to $\mathrm{PA}_{\eta}+\mathrm{A}_{\eta}^{T} \mathrm{P}=-\mathrm{I}_{m+n}$. The matrix $\mathrm{A}_{\eta}$ is upper triangular and its eigenvalues are the collection of those of $\mathrm{A}_{m}$ and $-1 / T_{\boldsymbol{f}} \mathrm{I}_{m}$. Hence, "P" can be arbitrarily reduced by choosing $\mathrm{A}_{m}, T_{\boldsymbol{f}}$ properly.

Remark 2. Note that, for given a controller tuning $\mathrm{A}_{m}$ and $T_{f}$, which satisfy the conditions of Theorem 1, the admissible delay is upper bounded by $h^{*}$. If the delay was larger, one would try to reduce "P" as indicated by Corollary 1 to keep the system stable, but doing so would have the opposite effect on $\left(\gamma_{x}+\gamma_{e}\right)$. Hence there is a maximum tolerable delay above which the system cannot be stabilized (this is well known for uncertain time-delay LTI systems).

Theorem 2. The system (1) controlled by (8), with an external disturbance $\mathrm{d}(t) \mathrm{f}=0$ satisfying Assumption 7, and a reference command satisfying Assumption 8, will be stable for some $\delta>0$ and any delay $0 \leq h \leq h^{*}$ if the

conditions in 1 heorem 1 hotd and $2 " \mathrm{P}_{2} "\left(\gamma_{0}+\rho \beta_{m}\right)$

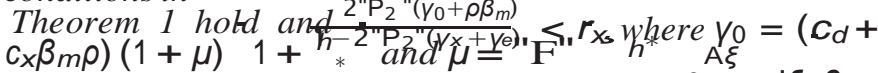
$\left.c_{x} \beta_{m} \rho\right)(1+\mu) 1+{ }_{T_{f}}$ and $\mu={ }_{0} e^{\mathrm{A} \xi} \mathrm{d} \xi \beta$. 
Proof. Considering external inputs, the term $Y_{0}$ in Lemma 1 does not vanish. Choosing the same Lyapunov function as in the proof of Theorem 1, the derivative along the new trajectories of (21) is given by

$$
\begin{aligned}
\dot{V}(\eta) & \left.\leq\left(2 " \mathrm{P}_{2} "\left(\gamma_{x}+\gamma_{e}\right)-1\right)\right) " \eta^{2} \\
& +2 " \mathrm{P}_{2} "\left(\gamma_{0}+\rho \beta_{m}\right) " \eta " \\
& \leq 0, \quad \text { if } \quad " \eta ">r_{\eta}
\end{aligned}
$$

with $r_{\eta}{ }^{3 / 4}{ }^{2 "} \mathrm{P}_{2}{ }^{\prime \prime}\left(\gamma_{0}+\rho \beta_{m}\right)$

$$
\frac{\mathrm{P}_{2}}{1-\mathrm{P}_{2} "\left(\chi_{x}+\gamma_{e}\right)} \text {. Note that } 1-2 " \mathrm{P}_{2} "\left(\gamma_{x}+\gamma_{e}\right)>0
$$
if the conditions of Theorem 1 are met. Then, according to (31), the region $\Omega_{\eta}=\left\{\eta \in \mathrm{R}^{n+m}: " \eta^{\prime} \leq r_{\eta}\right\}$ is positively invariant, which means that any trajectory starting from outside will eventually reach $\Omega_{\eta}$ and remain inside for all future time [33]. The parameter $r_{\eta}$ should be understood as how far from the origin the system is steered because of the inputs. Using

Theorem 4.18 in [33], if $r_{\eta}<r_{x}$, there exists a class $K L$ function $\gamma$ and a finite $T \geq 0$ for the initial state $" \eta(0) " \leq r_{x}$, such that the solution satisfies " $\eta(t) " \leq \gamma(" \eta(0) ", t), \forall 0 \leq t \leq T$ and $" \eta(t) " \leq r_{\eta}, \forall t \geq T$.

\section{Digital implementation}

Recall that the proposed control law is given by (8). Regarding the computation of $\mathrm{u}_{w}(t)$, using (15)-(17), the following expression for the UDE control action can be obtained (see Fig. 2)

$\mathrm{U}_{w}(s)=\left[\mathrm{I}-\mathrm{G}_{\boldsymbol{f}}(s)\right]^{-1} \mathrm{G}_{\boldsymbol{f}}(s) \mathrm{B}^{+}\left[\left(s \mathrm{I}-\mathrm{A}_{m}\right) \mathrm{Y}(s)+\mathrm{B}_{m} \mathbf{R}(s)\right]$,

which can be easily discretized and implemented in a digital micro-controller.

The other key variable to be computed is the predicted state $\hat{\mathrm{x}}\left(t+h_{1}\right)$. Its analytic expression is given in (4). The implementation of the distributed integral requires some attention [34], [35]. In this paper, the predictor is implemented in discrete-time form as in [28],

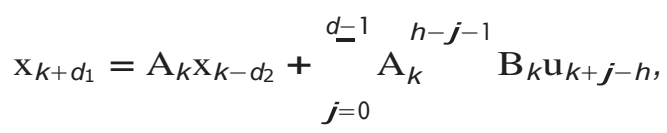

where $\left(\mathrm{A}_{k}, \mathrm{~B}_{k}\right)$ is a discretization of $(\mathrm{A}, \mathrm{B})$ and $d_{1}, d_{2}, d_{3} \in$ $\mathrm{N}$ are defined as $d_{1}=h_{1} / T_{s}, d_{2}=h_{2} / T_{s}, d=d_{1}+d_{2}$, being $T_{s}$ the discretization time.

To summarize the tuning procedure, four decisions are considered: the sample time $T_{s}$, the filter time constant $T_{\boldsymbol{f}}$, the prediction horizon $h$, and the desired reference model.

\section{Application to Quadrotor Aircraft}

In this section, the performance and robustness of the proposed strategy are illustrated through several simulations using a quadrotor model. These results are validated experimentally, first, in a Quanser laboratory platform, and later, in flight tests with a quadrotor prototype.

\section{A. Modeling of quadrotor systems}

A fairly accurate ${ }^{3}$ model of a quadrotor is given by the following set of nonlinear equations [4]

$$
\begin{aligned}
& \ddot{\varphi}(t)=I_{y}-I_{z} \dot{\theta}(t) \dot{\psi}(t)-J_{\Omega \dot{\theta}}(t)+u_{\varphi}(t-h),
\end{aligned}
$$

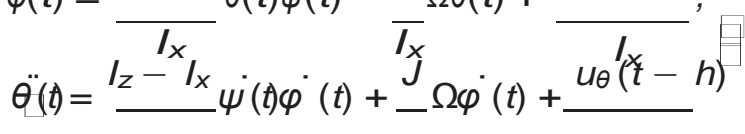

$$
\begin{aligned}
& \ddot{\psi}(t)=\frac{I_{x}-I_{y}}{I_{z}} \dot{\varphi}(t) \dot{\theta}(t)+\frac{I_{y}}{u_{\psi}(t-h)}, \\
& \ddot{z}(t)=\cos \varphi(t) \cos \theta(t) \frac{u_{z}(t-h)}{m}-g,
\end{aligned}
$$

where $\varphi, \theta$ and $\psi$ are the roll, pitch and yaw Euler angles, $I_{i}, i=\{x, y, z\}$ are the moments of inertia and $u_{i}, i=$ $\{\varphi, \theta, \psi\}$ are the input torques, all of them defined along the axes of a body-fixed reference frame, $z$ is the height, $m$ is the mass of the vehicle, $g$ is the gravity acceleration, $u_{z}$ is the input total thrust, $J$ is the inertia of the propellers and $\Omega$ is the sum of the angular velocities of the motors (taking the sign into account). An input delay $h$ is also considered. This delay may be caused by the communications with the Electronic Speed Controller of the motors, their response time and also because of the digital implementation of the control law.

The uncertainty in the rotational subsystem (32) satisfies Assumption 3 because it can be written in terms of (3) with

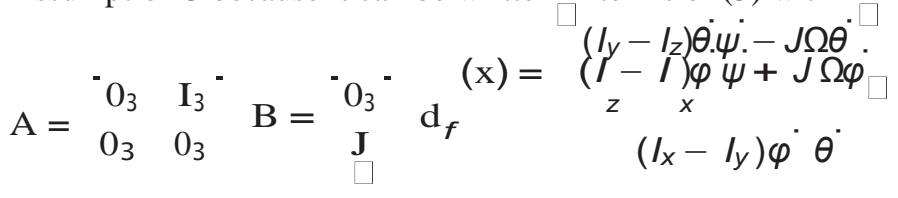

being $\mathrm{x}=[\varphi, \theta, \psi, \dot{\varphi}, \dot{\theta}, \dot{\psi}]^{T}$ the state vector, $\mathbf{J} 3 / 4$ $\operatorname{diag}\left\{I_{x}^{-1}, I_{y}^{-1}, I_{z}^{-1}\right\}$ and $\mathrm{u}=\left[u_{\varphi}, u_{\theta}, u_{\psi}\right]^{T}$. The matched uncertainty is locally bounded and vanishes at the origin.

Furthermore, its gradient is given by $\nabla \mathrm{d}_{\boldsymbol{f}}(x)=\left[\mathrm{O}_{3} \nabla_{12}\right]$ with

$$
\begin{array}{ccc}
0 & \left(I_{y}-I_{z}\right) \dot{\psi}-J \Omega & \left(I_{y}-I_{z}\right) \theta^{\cdot} \\
\nabla_{12} 3 / 4 \square\left(I_{z}-I_{x}\right) \dot{\psi}+J \Omega & 0 & \left(I_{z}-I_{x}\right) \dot{\varphi} \\
\left(I_{x}-I_{y}\right) \theta^{\cdot} & \left(I_{x}-I_{y}\right) \dot{0} & 0
\end{array}
$$

which is also locally bounded, thus satisfying the Assumption 4. Similarly, the height subsystem (33) satisfies the Assumption 3 because it can be written in terms of (3) with

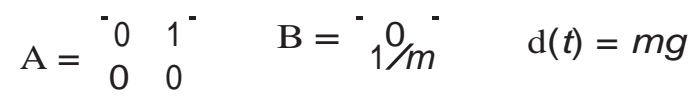

and $\mathrm{u}=u_{z} \cos \theta \cos \varphi$. Note that the disturbance $\mathrm{d}(t)$ is constant and satisfies Assumption 7, while in this case $\mathrm{d}_{f}(\mathrm{x}) \equiv 0$ thus satisfying Assumption 4. Regarding the finiteescape condition, the unforced system (32) is reduced to the Euler's equations that describe the free rotation of a rigid body. As the consequence of conservation of the energy and angular momentum, the solution to this set of equations is bounded in time [36]. Also, setting $u_{z}=0$ in (33) yields a linear equation.

\footnotetext{
${ }^{3}$ The rotational kinematic model used to derive (32) is linearized around the origin. Note that because of the singularity of the Euler representation, the linearization is only valid for $|\theta|<\pi / 2$.
} 

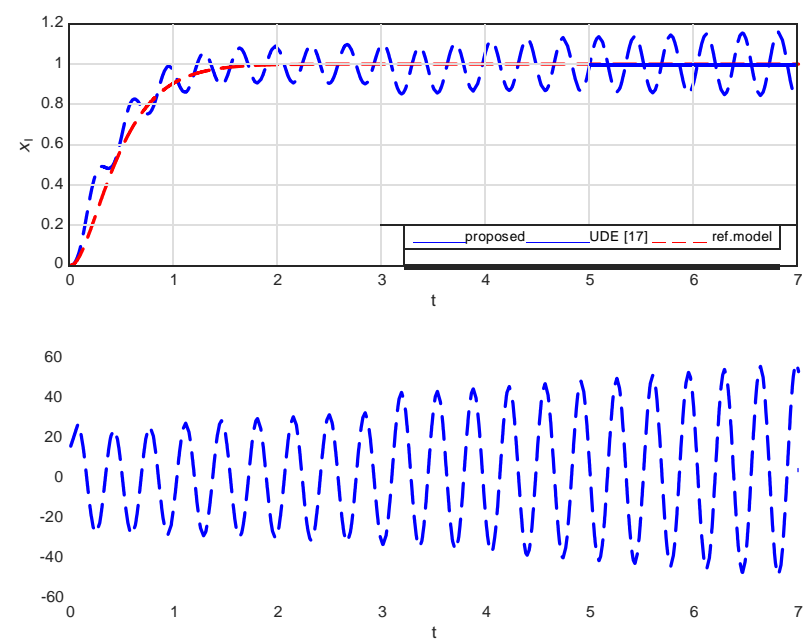

Fig. 3. Simulations comparing the original UDE [17] and the proposed strategy for a delay $h=70 \mathrm{~ms}$.

Therefore, there is not finite escape time and Assumption 6 holds.

Remark 3. From the arguments presented above, the proposed controller applied to quadrotor systems can only be proven to be locally stabilizing around the origin (in spite of uncertainties, external disturbances and input delay)

\section{B. Simulations}

For the sake of clarity, the performance and robustness are first illustrated using a SISO model. At the end of this section the control strategy is validated using the full nonlinear MIMO quadrotor model. The performance and robustness of the proposed strategy are illustrated next using one of the axes. Any of the equations in (32) can be seen as $\ddot{y}=b u(t-h)+w(t)$ or alternatively, in state-space form as

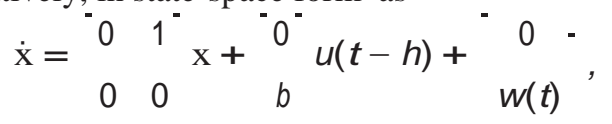

where $w(t)$ represents the interaction with the missing states, uncertainties in the parameter $b$ or external disturbances like wind gusts.

The controller is implemented as suggested in Section III-C, with $T_{s}=10 \mathrm{~ms}$ and the reference model given by

$$
\dot{\mathrm{x}}_{m}=\begin{array}{cc}
0 & 1 \\
-\omega_{c}^{2} & -2 \omega_{c}
\end{array} \mathrm{x}_{m}+{ }^{-} \omega_{c}^{2} \mathrm{y}^{\mathrm{ref}}(t),
$$

where $\omega_{c}$ is the desired closed-loop bandwidth. The parameters $T_{\boldsymbol{f}}$ and $h$ will be changed throughout this section.

1) Stability: Fig. 3 shows a comparison of the original UDE-based control without considering the delays [17] and the proposed strategy. The filter is tuned with $T_{\boldsymbol{f}}=0.1 \mathrm{~s}$ and the delay is $h=70 \mathrm{~ms}$. One can see how the proposed strategy can deal with that delay, ensures the matching of the reference model, and rejects input load disturbances.

2) Performance: The proposed strategy is compared with a PID controller $u(s)=K_{c} 1+\frac{T_{d s}}{\alpha T d s}+\frac{1}{T_{i} s} e(s)$, with a prefilter such that $e(s)=F_{r}(s) r(s)-y(s)$, with $F_{r}(s)=$
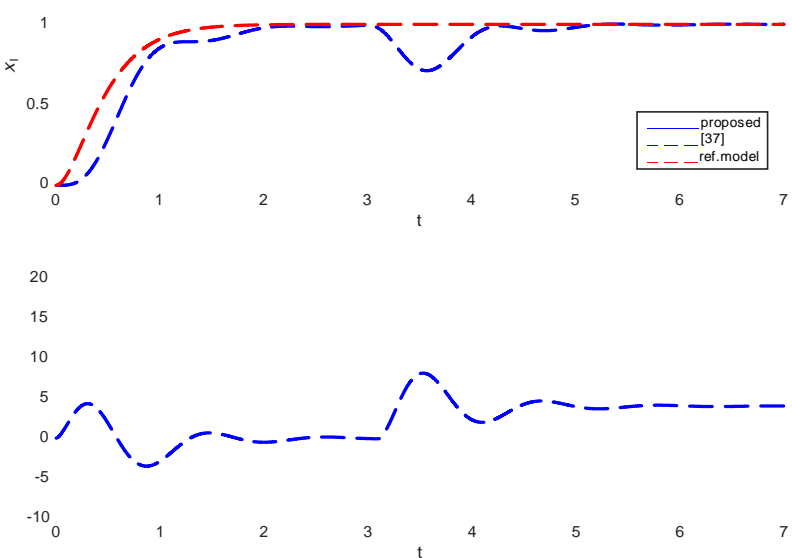

Fig. 4. Performance comparison with a conventional PID controller [37] with a delay $h=100 \mathrm{~ms}\left(T_{\mathbf{f}}=0.1 \mathrm{~s}\right)$
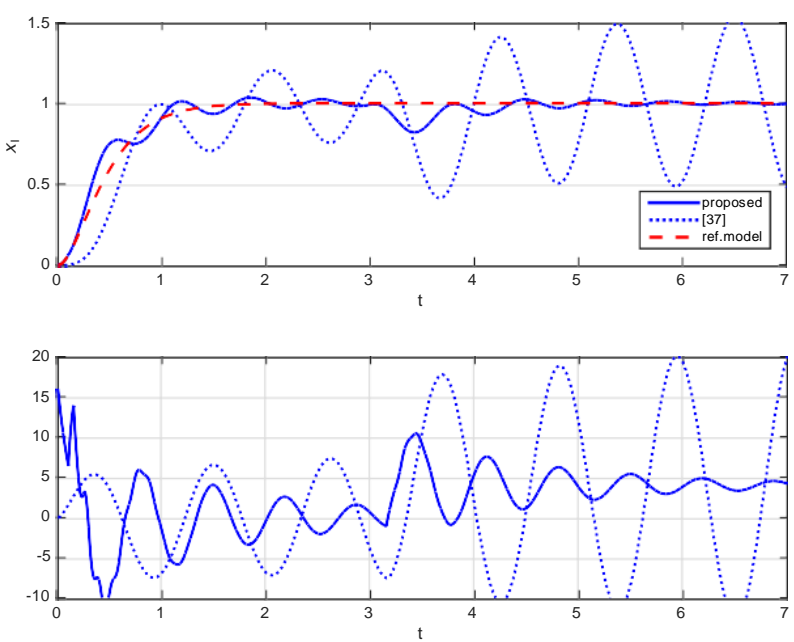

Fig. 5. Robustness conventional PID controller [37] with a $+50 \%$ delay error (filter tuned with $T_{\mathbf{f}}=0.1 \mathrm{~s}$ )

$\frac{2^{1}}{T_{d} T_{i S}+T_{i S+1}}$, and $K^{c}=\frac{0.13}{b h}, T_{i}=8.51 h, T_{d}=2.87 h$, $\alpha=0.1$, as suggested by [37]. These are, to the best of our knowledge, the simplest PID tuning rules for time delay systems in terms of performance. The comparison is shown in Fig. 4 for a delay $h=100 \mathrm{~ms}$, where it can be seen that the proposed control law outperforms the PID controller. In order to test the robustness against modeling errors in the delay, if an increment in the time delay of $50 \%$ is assumed, the proposal in [37] becomes unstable whereas the proposed strategy remains stable, as shown in Fig. 5.

Another simulation shows the influence of the filter time constant $T_{\boldsymbol{f}}$ in Fig. 6, where a wind disturbance is simulated between $t=2 \mathrm{~s}$ and $t=5 \mathrm{~s}$. The wind disturbance is simulated by passing a white noise signal through a low-pass filter. It can be clearly seen how the lower the $T_{\boldsymbol{f}}$, the better the disturbance rejection performance. It is also important that the choice of $T_{\boldsymbol{f}}$ does not affect the reference tracking performance, which is always desirable for an easier tuning.

3) Robustness: Robustness with respect to uncertainties in the delay $h$ is investigated. Fig. 7 shows different simulations 

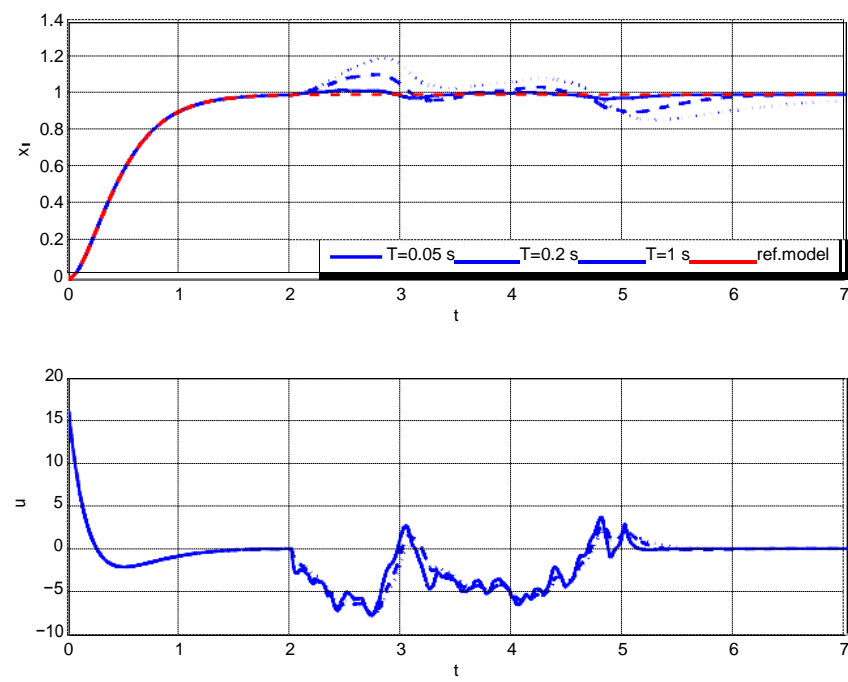

Fig. 6. Simulations showing the influence of the parameter $T_{\mathbf{f}}$ in external disturbance rejection performance
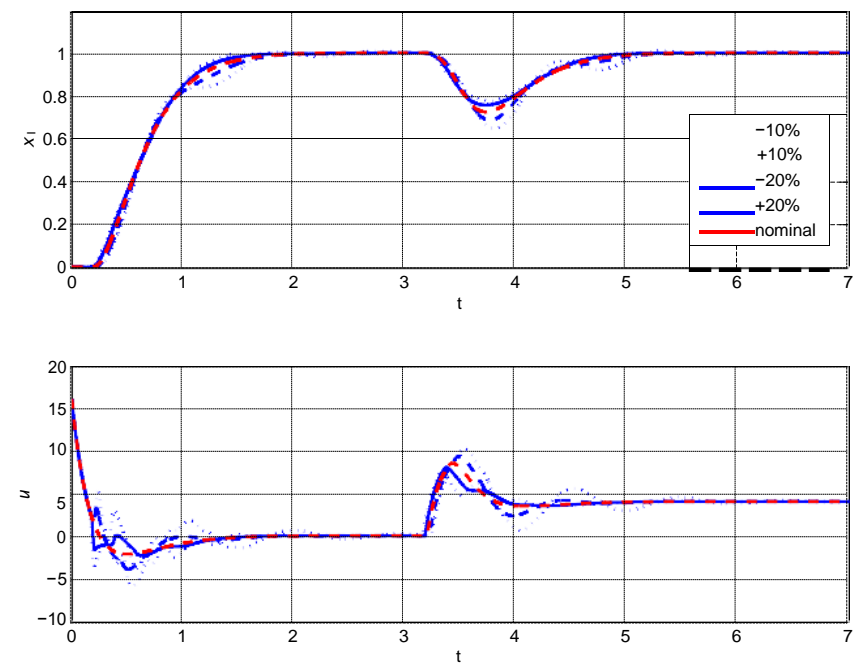

Fig. 7. Simulations showing robustness with respect to the uncertainty in the delay around a nominal value of $h=200 \mathrm{~ms}$ (filter tuned with $T_{\mathrm{f}}$ $=0.1 \mathrm{~s}$ )

with a percentage of uncertainty in the delay $h$ around a nominal value of $h=200 \mathrm{~ms}$.

4) Nonlinear multivariable model: Once the properties of the proposed strategy have been illustrated, the full control of a nonlinear quadrotor model in (32) is presented next. Comparing to the model in (7), it can be seen that for this particular case $\mathrm{x}=\left[\underline{\varphi}, \theta, \psi, \underline{\varphi}, \dot{\theta}^{*}, \psi^{*}\right]^{T}$,

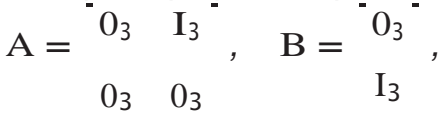

and

$$
\mathrm{f}(\mathrm{x}, t)=\dot{0}, \quad 0, \quad 0, \quad \dot{\theta} \dot{\psi}-\dot{\theta}, \quad \dot{\psi} \dot{\varphi}+\dot{\varphi}, \quad \dot{\varphi} \dot{\theta}^{T} .
$$

For simplicity, every axis is designed with the same closedloop dynamics, by specifying the following reference model

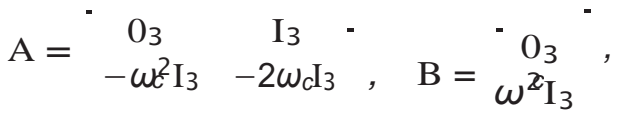
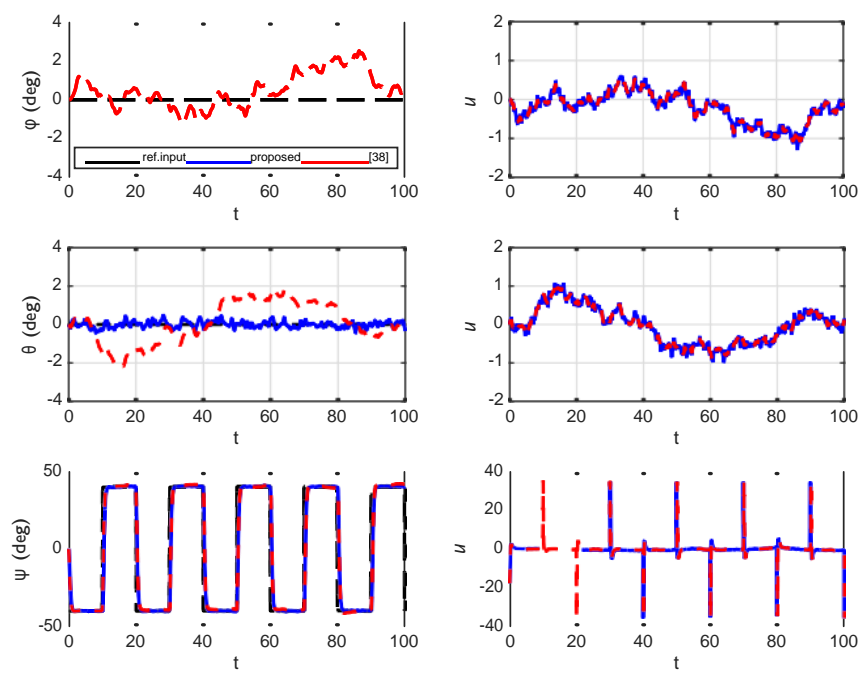

Fig. 8. Simulations showing hover flight and tracking performance in the yaw axis under the presence of wind disturbances (nonlinear MIMO system time delay $h=150 \mathrm{~ms}$ and filter tuned with $T_{\mathbf{f}}=0.1 \mathrm{~s}$ )

and selecting $\omega_{c}=5 \mathrm{rad} / \mathrm{s}$. A delay of $h=150 \mathrm{~ms}$ is considered and a filter time constant $T_{\boldsymbol{f}}=0.1 \mathrm{~s}$ is chosen. The simulation in Fig. 8 reproduces a real situation where the vehicle is perturbed by wind gusts and the goal is to keep it at hovering (zero reference in roll and pitch) while tracking a square reference signal in the yaw axis. The proposed controller is compared with that in [38], with $u_{x}(t)=-\sigma_{x_{1}}\left(k_{p} X\right)-\sigma_{i_{2}}\left(k_{d} \dot{x}\right)$ for each axis $x=\{\varphi, \theta, \psi\}$ and the saturation function is defined as

$$
\sigma_{b_{i}}(s)=\square \begin{array}{lr}
-b_{i}, & s<b_{i} \\
s, & -b_{i} \leq s \leq b_{i} \\
b_{i}, & s>b_{i}
\end{array} .
$$

The controller is tuned with $k_{p}=\omega_{c}^{2}$ and $k_{d}=2 \omega_{c}$, for the sake of comparison, and the saturation bounds are chosen as $b_{\varphi_{1}}=b_{\theta_{1}}=4, b_{\varphi_{2}}=b_{\theta_{2}}=5$ and $b_{\psi_{1}}=50, b_{\psi_{2}}=$ 60 . The bounds on the yaw axis have to be larger to allow good tracking performance. The benefit of this controller is that it allows to bound some of the states, thus limiting the size of the nonlinearities which depend on those states. However, in practice, small saturations make the system convergence very slow, leading to poor performance, as shown in Fig. 8, while the proposed controller is able to achieve satisfactory tracking and disturbance rejection in spite of the delay and nonlinearities.

\section{Experimental validation in a Quanser platform}

The 3D hover system shown in Fig. 9, which is a quadrotor with reduced degrees of freedom, is investigated. It consists of a quadrotor mounted on a 3 DOF pivot joint so that the body can freely rotate in roll, pitch and yaw. An Inertial Measurement Unit, the MPU6050 from InvenSense, is used to provide angular position and velocity measurements, using a Kalman filter algorithm which is run in an Arduino UNO micro-controller at $300 \mathrm{~Hz}$. There is a delay in the system which has mainly two sources: i) a measurement delay of about 20 ms due to the Kalman filter implementation, and ii 


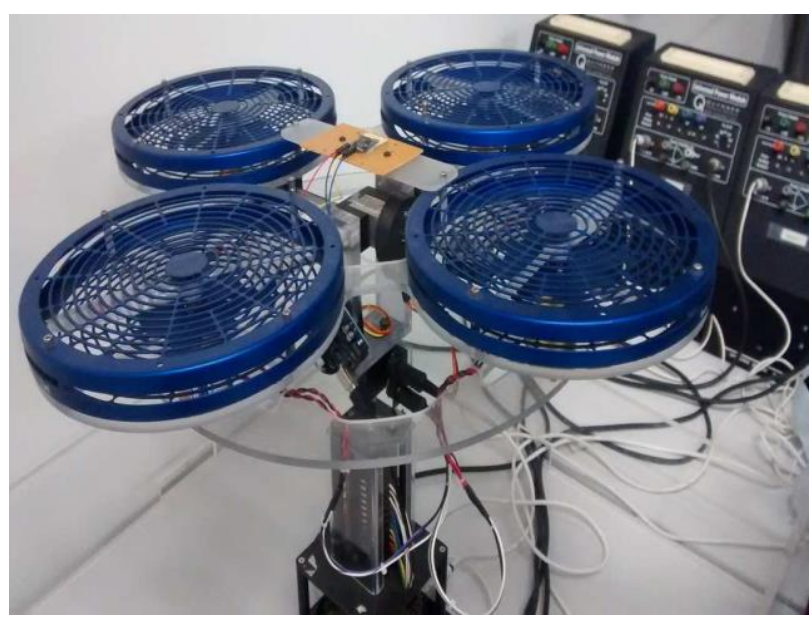

Fig. 9. Quanser experimental platform
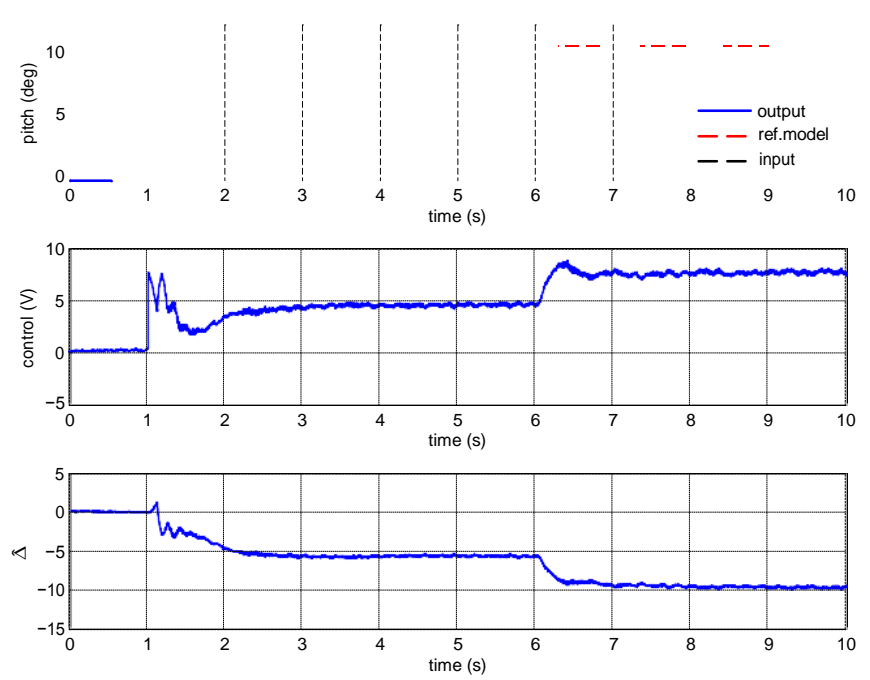

Fig. 10. Experimental results in a Quanser platform. Tracking and disturbance rejection performance of the proposed strategy for a delay $h=120 \mathrm{~ms}$.

the response time of the motors of about $100 \mathrm{~ms}$. The total delay of the system is thus estimated as $h=120 \mathrm{~ms}$.

For the controller synthesis, a single axis is modeled by a double integrator as in (35), with $b=0.13$. The controller is implemented in one of the axes as suggested in Section III-C with $T_{s}=3.3 \mathrm{~ms}, T_{\boldsymbol{f}}=0.2 \mathrm{~s}$, and the reference model (36), with desired closed-loop bandwidth $\omega_{c}=2.5 \mathrm{rad} / \mathrm{s}$.

The condition derived in Theorem 2 will be illustrated next for the Quanser platform. Consider the rotational dynamics of the quadrotor (32). The gradient of the matched uncertainty is given by (34), which satisfies Assumption 4 only locally. Because of the symmetry of the quadrotors, $I_{x}=I_{y}$, then $\boldsymbol{I} 3 / 4\left|\boldsymbol{I}_{z}-\boldsymbol{I}_{\boldsymbol{x}}\right|=\left|\boldsymbol{I}_{\boldsymbol{y}}-\boldsymbol{I}_{z}\right|$. Also, in the Quanser platform, the motors are bidirectional, and they are driven such that $\Omega_{2}=-\Omega_{1}$ and $\Omega_{3}=-\Omega_{4}$. Hence the gyroscopic torque vanishes because ideally $\Omega 3 / 4-{ }_{i=1} \Omega_{i}=0$. Hence, from (34), the gradient of the unknown dynamics is bounded by

$$
" \nabla d_{f}(x) " \leq \max { }^{\prime} / \dot{\psi}, I^{-}\|\dot{\theta}\|+\dot{\varphi}^{-} \leq / x^{\prime \prime} 3 / 4 c_{x} .
$$
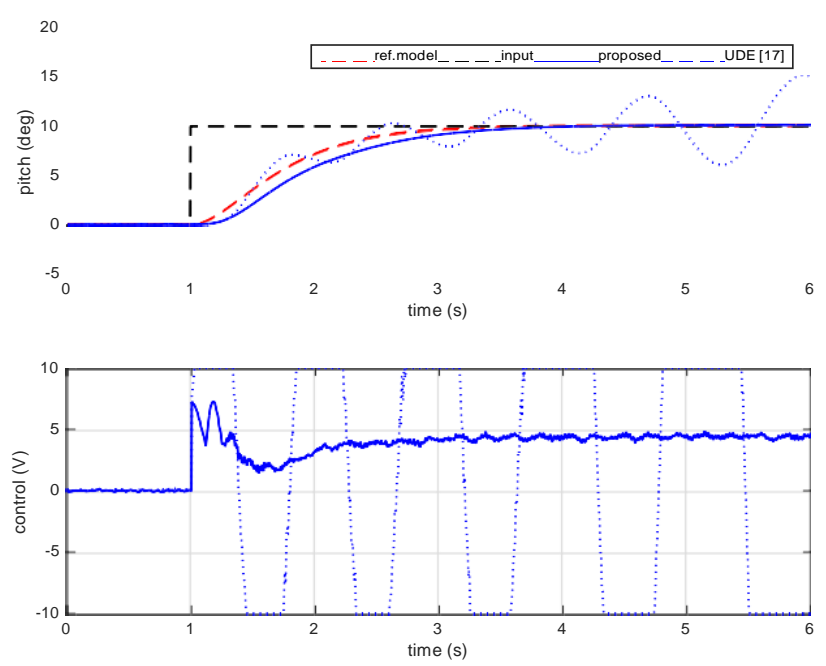

Fig. 11. Experimental results in a Quanser platform. Stability comparison of the original UDE [17] and the proposed strategy for a delay $h=120 \mathrm{~ms}$.

Therefore, one has that $c_{x}=I r_{x}, \forall x \in D$. In order to illustrate Theorem 2, let us choose a region with $r_{x}=3$ (which fulfills ${ }^{4}$ Theorem 1 because 2 " $\left.\mathrm{P}_{2} "\left(y_{x}+\gamma_{e}\right) \approx 0.82<1\right)$ in which the maximum norm of the gradient is $c_{X}=I r_{x} \approx 0.16$. As pointed out in Theorem 2, it is possible to find the maximum input command that ensures stability by solving $r_{\eta}=r_{x}$. Using equation (31), assuming $C_{d}=0$ and solving for $\rho$ yields

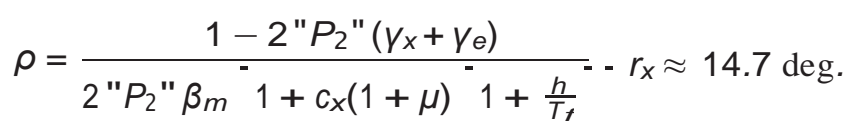

The next experiment complies with these bounds because the commanded angle is less than $14.7 \mathrm{deg}$ and the system satisfies $" x(0) "+" e(0) "<3$ as it starts from the origin. Note that $e(0)=0$ because the observer starts also from zero.

The results of the first experiment are shown in Fig. 10. A step input reference of $10^{\circ}$ is applied at $t=0$ followed by a step input disturbance simulated by software at $t=6$ s. It is possible to see how the response of the system matches that of the reference model. The disturbance is also rapidly rejected, achieving zero steady-state tracking error. It is also interesting to look how the estimation of the unknown dynamics converges to some value, which is what keeps the system precisely at $10^{\circ}$. If the system was an ideal double integrator, this value would be zero. But this is not the case and the observer is able to estimate that uncertainty. Note also how the input disturbance is also estimated after it is applied at $t=6 \mathrm{~s}$.

As it has been discussed above, the original UDE reduces the delay margin of the system drastically. In order to illustrate this fact, the same experiment as before is carried out using the original UDE-based control [17], and keeping the same tuning parameters. Both responses are shown in Fig. 11, where it can be seen that the original UDE is clearly unstable. 


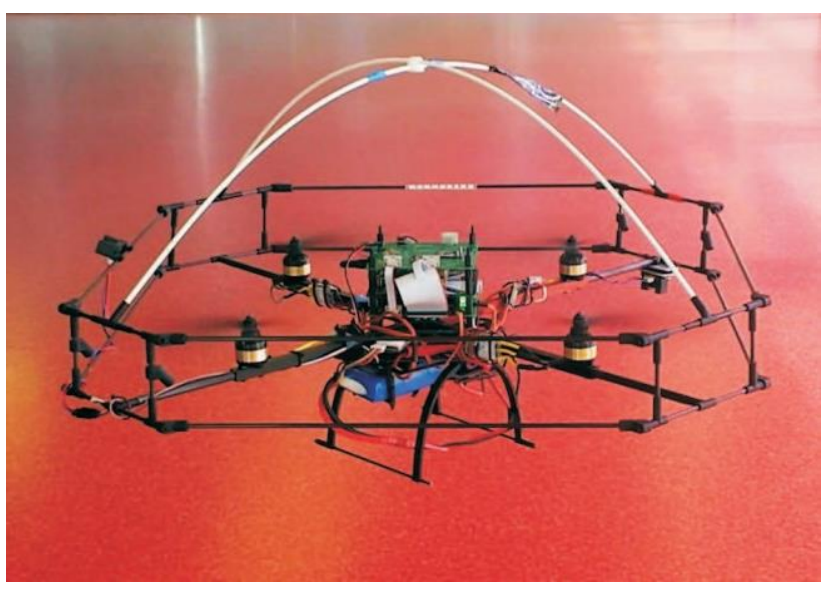

Fig. 12. Quadrotor prototype used in experiments

TABLE I

Controller Parameters in Flight Tests

\begin{tabular}{c|cccc}
\hline \hline & $b\left[\mathrm{deg} / \mathrm{s}^{2}\right]$ & $\omega_{\mathrm{c}}[\mathrm{rad} / \mathrm{s}]$ & $T_{\mathbf{f}}[\mathrm{s}]$ & $h[\mathrm{~ms}]$ \\
\hline$\varphi$ & 1500 & 2.5 & 0.6 & 25 \\
$\theta$ & 1500 & 2.5 & 0.6 & 25 \\
$\psi$ & 1000 & 4 & 1 & 25 \\
$z$ & 3000 & 2.5 & 0.6 & 250 \\
\hline \hline
\end{tabular}

\section{Experimental validation in flighttests}

There are some handicaps to overcome in real flights, e.g., large model uncertainties, measurement noise, flapping and ground effects, wind gusts, etc. The quadrotor prototype used for the experiments has a distance of $41 \mathrm{~cm}$ between rotors, weighting about $1.3 \mathrm{~kg}$ without battery. The basic hardware consists of a MikroKopter frame, YGE 25i electronic speed controllers, RobbeRoxxy 2827-35 brushless motors and $10 \times 4.5$ plastic propellers. All the computations are carried out on-board at $400 \mathrm{~Hz}$ using an Igep v2 board running Xenomai real-time operating system. In this system, the delays in the control loop come from: i) the Kalman filter algorithm which introduces a small delay due in the measurements, and ii) the response time of the motors drivers.

For the controller synthesis, each axis is modeled by a double integrator as in (35). The controller is implemented with a sample time $T_{s}=2.5 \mathrm{~ms}$ and a reference model as in (36) is proposed. The controller tuning is shown in Table I.

The first experiment consists of applying yaw and height step references during stationary flight (roll and pitch references set to zero). The result of this experiment is shown in the Fig. 13. One can see how the roll and pitch angles are kept very close to zero. Their root mean squared errors are 0.6 deg and $0.7 \mathrm{deg}$ for roll and pitch, respectively. The tracking performance of the yaw angle is very good. The performance in the height control is also remarkable, because it is more

\footnotetext{
${ }^{4} \mathrm{~A}$ quadrature algorithm in Matlab has been used to compute the term ${ }_{0}^{\mathrm{h}} e^{\mathrm{A} \xi} \mathrm{d} \xi$
}
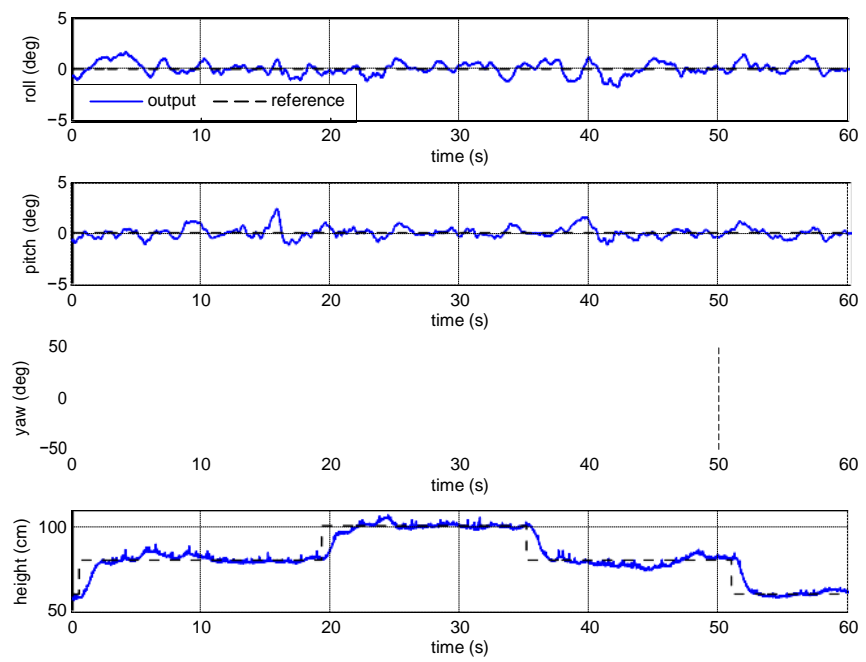

Fig. 13. Tracking performance in a real flight.
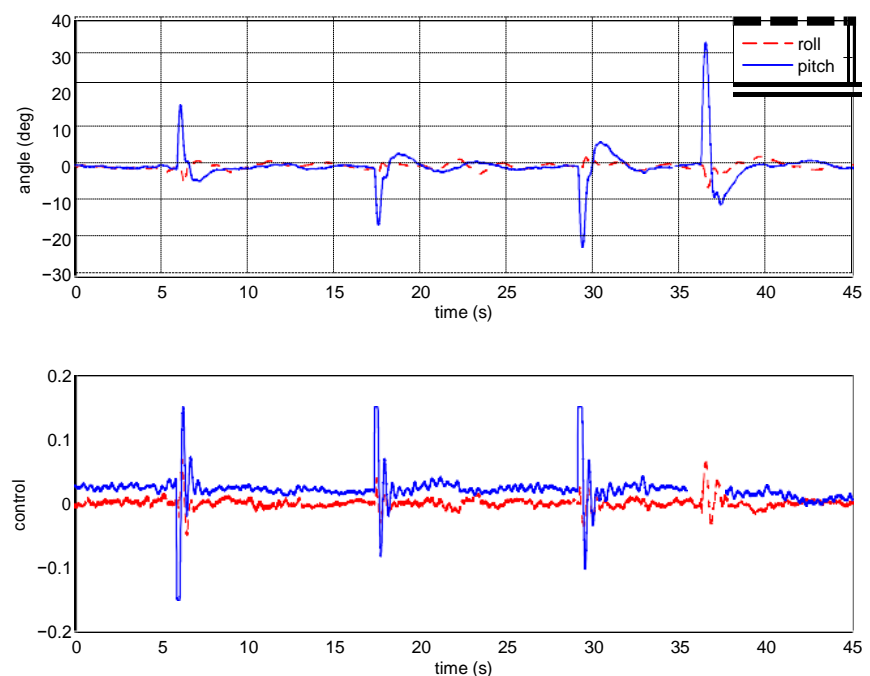

Fig. 14. Disturbance rejection in a real flight. A video of this experiment is available at https://youtu.be/AaCOYglzBao .

challenging due to the large delay of the ultrasonic sensor and the huge mass of the vehicle.

In the second experiment, disturbances are applied to the quadrotor in stationary flight. These disturbances are generated by hitting the vehicle in the pitch axis. The result of this experiment is shown in the Fig. 14 where it can be seen that the vehicle recovers successfully. It is remarkable that the quadrotor is deviated more than 30 deg from its equilibrium point and yet it remains stable.

\section{CONCLUSIONS}

In this paper, a control strategy based on a disturbance observer has been developed to deal with systems with input and output delays. A predictor is adopted to counteract the effect of the delay in the feedback loop, while a modified uncertainty and disturbance estimator (UDE) is used to compensate for model uncertainties and reject input disturbances. The proposed strategy not only is applicable to systems with 
large delays, but it also results in outstanding performance when applied to systems with small delays, inherent to any digital implementation. These results have been demonstrated with extensive simulations and experiments.

\section{ACKNOWLEDGMENT}

The authors would like to thank the reviewers for their insightful and valuable comments, which have helped the authors significantly improve the quality of this paper

\section{REFERENCES}

[1] P. Castillo, A. Dzul, and R. Lozano, "Real-time stabilization and tracking of a four-rotor mini rotorcraft," IEEE Trans. Control Syst. Technol., vol. 12, no. 4, pp. 510-516, Jul. 2004.

[2] R. Mahony, V. Kumar, and P. Corke, "Multirotor aerial vehicles: Modeling, estimation, and control of quadrotor," IEEE Robotics \& Automation Magazine, vol. 19, no. 3, pp. 20-32, Sep. 2012.

[3] S. Bouabdallah and R. Siegwart, "Full control of a quadrotor," in IEEE/RSJ Int. Conf. on. Intelligent Robots and Systems. IEEE, 2007, pp. $153-158$.

[4] S. Bouabdallah, A. Noth, and R. Siegwart, "PID vs LQ control techniques applied to an indoor micro quadrotor," in IEEE/RSJ Int. Conf. on. Intelligent Robots and Systems. IEEE, 2004, pp. 2451-2456.

[5] P. Castillo, R. Lozano, and A. Dzul, "Stabilization of a mini rotorcraft with four rotors," IEEE Control Systems Magazine, vol. 25, no. 6, pp. 45-55, Dec. 2005.

[6] A. Sanchez, P. Garcia, P. C. Garcia, and R. Lozano, "Simple realtime stabilization of vertical takeoff and landing aircraft with bounded signals," Journal of Guidance, Control, and Dynamics, vol. 31, no. 4, pp. 1166-1176, Jul. 2008.

[7] H. Lim, J. Park, D. Lee, and H. J. Kim, "Build your own quadrotor: Open-source projects on unmanned aerial vehicles," IEEE Robotics \& Automation Magazine, vol. 19, no. 3, pp. 33-45, Sep. 2012.

[8] T. Tomic, K. Schmid, P. Lutz, A. Domel, M. Kassecker, E. Mair, I. L. Grixa, F. Ruess, M. Suppa, and D. Burschka, "Toward a fully autonomous UAV: research platform for indoor and outdoor urban search and rescue," IEEE Robotics \& Automation Magazine, vol. 19, no. 3, pp. 46-56, Sep. 2012.

[9] B. Zheng and Y. Zhong, "Robust attitude regulation of a 3-DOF helicopter benchmark: theory and experiments," IEEE Trans. Ind. Electron., vol. 58 , no. 2 , pp. 660-670, Feb. 2011

[10] S. Islam, P. X. Liu, and A. El Saddik, "Robust control of four rotor unmanned aerial vehicles with disturbance uncertainty," IEEE Trans. Ind. Electron., vol. 62, no. 3, pp. 1563 - 1571, Mar. 2015.

[11] B. Zhao, B. Xian, Y. Zhang, and X. Zhang, "Nonlinear robust adaptive tracking control of a quadrotor UAV via immersion and invariance methodology," IEEE Trans. Ind. Electron., vol. 62, no. 5, pp. 2891 2902, May. 2015.

[12] G. Hoffmann, H. Huang, S. Waslander, and C. Tomlin, Quadrotor Helicopter Flight Dynamics and Control: Theory and Experiment. AIAA, 2016/08/15 2007.

[13] S. Waslander and C. Wang, Wind Disturbance Estimation and Rejection for Quadrotor Position Control. AIAA, 2016/08/15 2009.

[14] W. Dong, G.-Y. Gu, X. Zhu, and H. Ding, "High-performance trajectory tracking control of a quadrotor with disturbance observer," Sensors and Actuators A: Physical, vol. 211, pp. 67-77, May. 2014.

[15] K. Lee, J. Back, and I. Choy, "Nonlinear disturbance observer based robust attitude tracking controller for quadrotor UAVs," Int. J. Control Autom. and Systems, vol. 12, no. 6, pp. 1266-1275, Dec. 2014.

[16] W. Sun, Z. Zhao, and H. Gao, "Saturated adaptive robust control for active suspension systems," IEEE Trans. Ind. Electron., vol. 60, no. 9, pp. 3889-3896, Sep. 2013.

[17] Q.-C. Zhong and D. Rees, "Control of uncertain LTI systems based on an uncertainty and disturbance estimator," Journal of Dynamic Systems, Measurement, and Control, vol. 126, no. 4, pp. 905-910, Dec. 2004.

[18] Q.-C. Zhong, A. Kuperman, and R. Stobart, "Design of UDE-based controllers from their two-degree-of-freedom nature," International Journal of Robust and Nonlinear Control, vol. 21, no. 17, pp. 1994-2008, Nov. 2011.

[19] J. Yao, Z. Jiao, and D. Ma, "Adaptive robust control of DC motors with extended state observer," IEEE Trans. Ind. Electron., vol. 61, no. 7, pp. 3630-3637, Jul. 2014.
[20] K. Ohishi, M. Nakao, K. Ohnishi, and K. Miyachi, "Microprocessorcontrolled DC motor for load-insensitive position servo system," IEEE Trans. Ind. Electron., vol. IE-34, no. 1, pp. 44-49, Feb. 1987.

[21] J. Han, "From PID to active disturbance rejection control," IEEE Trans. Ind. Electron., vol. 56, no. 3, pp. 900-906, Mar. 2009.

[22] J. P. Kolhe, M. Shaheed, T. Chandar, and S. Talole, "Robust control of robot manipulators based on uncertainty and disturbance estimation," International Journal of Robust and Nonlinear Control, vol. 23, no. 1, pp. 104-122, Jan. 2013

[23] B. Zhu, H. H.-T. Liu, and Z. Li, "Robust distributed attitude synchronization of multiple three-DOF experimental helicopters," Control Engineering Practice, vol. 36, pp. 87-99, Mar. 2015.

[24] B. Ren, Q.-C. Zhong, and J. Chen, "Robust control for a class of nonaffine nonlinear systems based on the uncertainty and disturbance estimator," IEEE Trans. Ind. Electron., vol. 62, no. 9, pp. 5881-5888, Sep. 2015.

[25] A. Kuperman and Q.-C. Zhong, "Ude-based linear robust control for a class of nonlinear systems with application to wing rock motion stabilization," Nonlinear Dynamics, vol. 81, no. 1-2, pp. 789-799, Jul. 2015.

[26] Q.-C. Zhong, Robust control of time-delay systems. Springer Science \& Business Media, 2006.

[27] R. Sanz, P. Garcia, P. Castillo, and P. Albertos, "Time-delay compensation using inertial measurement sensors for quadrotor control systems," in Information Fusion (FUSION), 2014 17th International Conference on. IEEE, 2014, pp. 1-6.

[28] R. Lozano, P. Castillo, P. Garcia, and A. Dzul, "Robust prediction-based control for unstable delay systems: application to the yaw control of a mini-helicopter," Automatica, vol. 40, no. 4, pp. 603-612, Apr. 2004.

[29] M. Krstic, "On compensating long actuator delays in nonlinear control," IEEE Trans. Autom. Control, vol. 53, no. 7, pp. 1684-1688, Aug. 2008. [30] M. Krstic, "Compensation of infinite-dimensional actuator and sensor dynamics," IEEE Control Syst. Mag., vol. 30, no. 1, pp. 22-41, Feb. 2010.

[31] D. Schmidt, Modern flight dynamics. McGraw-Hill Higher Education, 2012.

[32] H. Trinh and M. Aldeen, "On robustness and stabilization of linear systems with delayed nonlinear perturbations," IEEE Trans. Autom. Control, vol. 42, no. 7, pp. 1005-1007, Jul. 1997.

[33] H. K. Khalil and J. Grizzle, Nonlinear systems. Prentice hall New Jersey, 1996.

[34] Q.-C. Zhong, "On distributed delay in linear control laws-part I: discretedelay implementations," IEEE Trans. Autom. Control, vol. 49, no. 11, pp. 2074-2080, Nov. 2004.

[35] Q.-C. Zhong, "On distributed delay in linear control laws-part II: rational implementations inspired from the $\delta$-operator," IEEE Trans. Autom. Control, vol. 50, no. 5, pp. 729-734, May. 2005.

[36] G. R. Fowles and G. L. Cassiday, Analytical mechanics. Cengage Learning, 2004

[37] A. Ali and S. Majhi, "PID controller tuning for integrating processes," ISA Transactions, vol. 49, no. 1, pp. 70-78, Jan. 2010.

[38] G. Sanahuja, P. Castillo, and A. Sanchez, "Stabilization of n integrators in cascade with bounded input with experimental application to a VTOL laboratory system," International Journal of Robust and Nonlinear Control, vol. 20, no. 10, pp. 1129-1139, Jul. 2010. 